\title{
Excess purine degradation caused by an imbalance in the supply of adenosine triphosphate in patients with congestive heart failure
}

Ichiro Hisatome, Riichiro Ishiko, Hiroyuki Miyakoda, Makoto Saito, Hideyuki Kitamura, Toru Kinugawa, Mikihisa Kobayashi, Hiroshi Kotake, Hiroto Mashiba, Ryoichi Sato

\begin{abstract}
To evaluate purine degradation in patients with congestive heart failure concentrations of serum hypoxanthine, lactate, and noradrenaline were measured before and after submaximal treadmill exercise in 12 patients with chronic congestive heart failure and nine healthy volunteers. In four patients the concentration of hypoxanthine was significantly higher than in the controls or in the remaining eight patients with congestive heart failure. Venous lactate and noradrenaline in the four patients with high concentrations of hypoxanthine were also significantly higher than those in the eight patients with normal concentrations of hypoxanthine. Patients who responded normally were also more likely to have been treated with vasodilators and angiotensin converting enzyme inhibitors. Exercise induced arrhythmias were more common in the patients with high concentrations of hypoxanthine.
\end{abstract}

These results suggest that the excess purine degradation in patients with congestive heart failure might be the result of a "relative" disturbance in the supply of adenosine triphosphate caused by the shift of cellular metabolism from aerobic glycolysis to anaerobic glycolysis during submaximal exercise and that hypoxanthine (a substrate for xanthine oxidase and a source of free radicals) was increased after submaximal exercise in some patients with congestive heart failure.

Exercise induces the breakdown of adenosine triphosphate (ATP) and an associated increase in adenosine diphosphate (ADP) and adenosine monophosphate (AMP) in the muscle. ${ }^{1}$ Cells try to maintain a high energy charge even if the concentration of ATP falls and the energy level decreases. ${ }^{2}$ The formula for energy charge $(\mathrm{EC}): \mathrm{EC}=\mathrm{ATP}+0.5 \mathrm{ADP}$ $A M P+A D P+A T P$, indicates that a reduction in the concentration of AMP via activation of AMP deaminase, which accelerates purine degradation, will keep the energy charge high during ATP deficiency. The cells, however, can meet energy demands by producing ATP through aerobic glycolysis or creatinine phosphate. The ATP produced blocks AMP deaminase allosterically and increases purine degradation. Excess purine degradation in the muscle was reported in glycogen storage disease of types III, V, and VII. ${ }^{3}$ In these patients an "absolute" disturbance in the supply of ATP from glycogenolysis or glycolysis during exercise facilitated the breakdown of ATP and/or increased ADP and AMP, resulting in an increase in hypoxanthine-that is, urate precursors-by activation of AMP deaminase and accelerated purine degradation. In patients with hyperthyroidism ${ }^{4}$ we found that a "relative" disturbance of ATP supply - that is the demand for ATP exceeded its supplyalso induced the excess purine degradation in skeletal muscle.

We now report excess purine degradation induced by submaximal treadmill exercise in some patients with congestive heart failure. We believe that the increase in serum hypoxanthine in these patients may have been related to a "relative" disturbance in the supply of ATP during submaximal exercise which may be partly the result of a shift in cellular metabolism in the muscles from aerobic glycolysis to anaerobic glycolysis.

\section{Patients and methods}

We studied 12 patients with chronic, stable, congestive heart failure class II and nine healthy volunteers. Subjects exercised on a treadmill according to the modified Bruce protocol. They stopped exercising when they achieved $85 \%$ of their calculated maximal predicted heart rate without becoming short of breath or fatigued.

To investigate purine degradation we collected venous blood specimens through a catheter in the antecubital vein to measure serum lactate, hypoxanthine, and noradrenaline, creatinine, and creatine kinase after 15 minutes' bed rest, immediately after the end of exercise, and 5 and 10 minutes after the end of an exercise test with 3 minute stages. Blood lactate was measured enzymatically. Hypoxanthine and noradrenaline were measured by high performance liquid chromatography.

\section{Results}

Figure A shows the changes in hypoxanthine from basal concentration-that is the concentration at rest-in four patients with congestive heart failure after a submaximal treadmill exercise test. The shaded area shows the mean (2 SD) increase in plasma hypoxanthine in nine healthy individuals after a submaximal exercise test. In four patients with congestive heart failure plasma concentrations of hypox- 

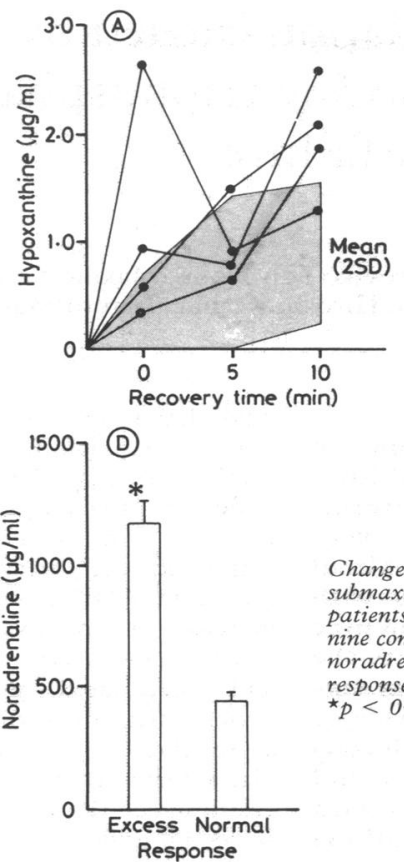
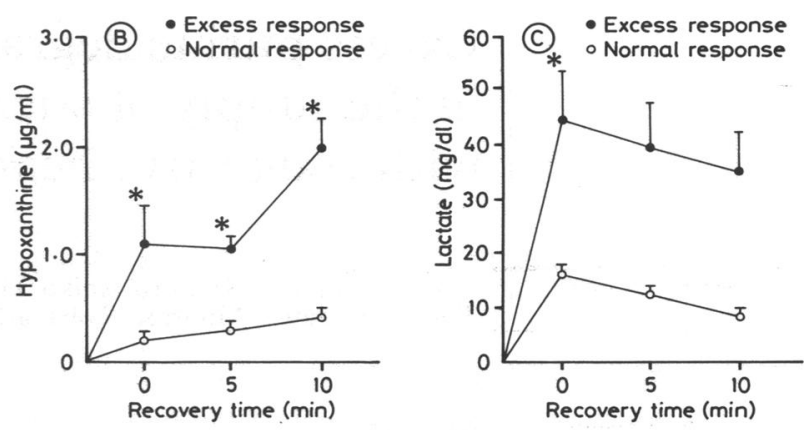

Changes in plasma concentrations of hypoxanthine, lactate, and noradrenaline after submaximal exercise. (A) Increase in hypoxanthine concentration after exercise in four patients with congestive heart failure. The shaded area indicates the mean (2SD) in nine controls. Mean (1 SD) increase in plasma hypoxanthine (B), lactate (C), and noradrenaline (D) concentrations after exercise in the four patients with an abnormal response and the eight with a normal response. ${ }^{\star} p<0.01$.

Clinical characteristics of patients with congestive heart failure in patients with an abnormal hypoxanthine response and a normal response

\begin{tabular}{|c|c|c|c|c|c|c|}
\hline \multirow[b]{2}{*}{ Group } & \multirow[b]{2}{*}{ Patient } & \multirow[b]{2}{*}{ Age and sex } & \multirow[b]{2}{*}{ Diagnosis } & \multirow[b]{2}{*}{ Treatment } & \multicolumn{2}{|l|}{ Arrhythmia } \\
\hline & & & & & Rest & Exercise \\
\hline \multirow{3}{*}{$\begin{array}{l}\text { Raised plasma } \\
\text { hypoxanthine: }\end{array}$} & 1 & $61 \mathrm{~F}$ & OMI & Dig, ca, diu & $(-)$ & \multirow{3}{*}{$\begin{array}{l}\operatorname{SVPC}(+) \\
\operatorname{VPC}(+) \\
\operatorname{SVPC}(+) \\
\operatorname{SVPC}(+) \\
\operatorname{VPC}(+) \\
(-)\end{array}$} \\
\hline & $\begin{array}{l}2 \\
3\end{array}$ & $\begin{array}{l}55 \mathrm{~F} \\
40 \mathrm{M}\end{array}$ & $\begin{array}{l}\text { MS } \\
\text { MR }\end{array}$ & $\stackrel{(-)}{(-)}$ & $\stackrel{(二)}{(-)}$ & \\
\hline & 4 & $51 \mathrm{M}$ & OMI & Dig & $(-)$ & \\
\hline \multirow{2}{*}{$\begin{array}{l}\text { Normal plasma } \\
\text { hypoxanthine: }\end{array}$} & 5 & $56 \mathrm{~F}$ & MSR & Dig & $\operatorname{SVPC}(+)$ & $\operatorname{SVPC}(+)$ \\
\hline & $\begin{array}{r}6 \\
7 \\
8 \\
9 \\
10 \\
11 \\
12\end{array}$ & $\begin{array}{l}74 \mathrm{~F} \\
50 \mathrm{M} \\
64 \mathrm{~F} \\
62 \mathrm{~F} \\
59 \mathrm{~F} \\
40 \mathrm{~F} \\
44 \mathrm{M}\end{array}$ & $\begin{array}{l}\text { MSR + ASR } \\
\text { OMI } \\
\text { ASR + MSR } \\
\text { HTH } \\
\text { IHD } \\
\text { MS, AR } \\
\text { ASD }\end{array}$ & $\begin{array}{l}\text { Dig, diu, nitrate } \\
\text { Dig, diu, nitrate, ACEI } \\
\text { Dig, diu } \\
\text { Ca } \\
\text { Dig, diu, nitrate, ACEI } \\
\text { Dig, diu } \\
(-)\end{array}$ & $\begin{array}{l}\operatorname{VPC}(+) \\
(-) \\
\operatorname{VPC}(+) \\
\operatorname{VPC}(+) \\
(-) \\
\operatorname{SVPC}(+)\end{array}$ & $\begin{array}{l}\operatorname{VPC}(+) \\
\operatorname{VPC}(+) \\
\operatorname{VPC}(+) \\
\operatorname{VPC}(-) \\
(-) \\
(-) \\
(-)\end{array}$ \\
\hline
\end{tabular}

ACEI, ACE inhibitor; AR, aortic regurgitation; ASR, aortic stenosis and regurgitation; ASD, atrial septal defect; Ca, calcium antagonist; Dig, digitalis; diu, diuretics; HTH, hypertensive heart disease; IHD, ischaemic heart disease; MR, mitral antagonist; Dig, digitalis; diu, diuretics; HTH, hypertensive heart disease; IHD, ischaemic heart disease; MR, mitral
regurgitation; MS, mitral stenosis; MSR, mitral stenosis and regurgitation; OMI, old myocardial infarction; SVPC, supraventricular premature contraction; VPC, ventricular premature contraction.

anthine were considerably increased after submaximal exercise. In the remaining eight patients they were not significantly different from concentrations in the healthy volunteers (fig B). In the four patients with increased concentrations of serum hypoxanthine and the eight with normal concentrations the exercise test was stopped when they reached $85 \%$ of the maximum predicted heart rate without symptoms. The mean (SD) duration of exercise was similar in both groups (7.0 (1.3) minutes in patients with a high concentration and $6.9(1.7)$ minutes in patients with a normal concentration of hypoxanthine. Figures $C$ and $D$ show that increases in lactate and noradrenaline from basal concentrations were significantly higher in patients with high hypoxanthine concentrations after submaxi- mal treadmill exercise than in those with normal concentrations.

The table shows the clinical characteristics of all the patients with congestive heart failure. The range of diagnoses was similar in patients with an increased concentration of serum hypoxanthine after submaximal exercise and those without, and the mean ejection fraction of the left ventricle in the two groups was similar too $(54.3 \%$ and $53.2 \%$ respectively). Only one of the four patients with high hypoxanthine concentrations was taking vasodilators and none of them was taking an angiotensin converting enzyme inhibitor. However, four of the eight patients with a normal hypoxanthine response were taking a vasodilator and two others were taking an angiotensin converting enzyme inhibitor. 
When we remeasured the hypoxanthine response in patient 2 after percutaneous balloon dilatation of the mitral valve the serum hypoxanthine concentration was normal.

Three of the four patients with high concentrations of serum hypoxanthine had exercise induced arrhythmias whereas only one of the eight patients with a normal hypoxanthine response had exercise induced arrhythmia.

\section{Discussion}

We showed that the plasma concentration of hypoxanthine was increased after submaximal treadmill exercise in some patients with congestive heart failure. Concentrations of blood lactate and plasma noradrenaline were also increased in these patients. Exercise increased plasma hypoxanthine in glycogen storage disease types III, V, VII $^{3}$ and hyperthyroidism ${ }^{4}$ and this increase in hypoxanthine was associated with excess purine degradation. These findings suggested that hypoxanthine was a good index of purine degradation via the purine nucleotide cycle, because increased AMP deamination via activation of AMP deaminase could accelerate the subsequent breakdown of inosine monophosphate to inosine, thereby leading to the formation of hypoxanthine. So the abnormal increase in plasma hypoxanthine in four of our patients with congestive heart failure may reflect the activation of the purine nucleotide cycle in the skeletal muscle. Theoretically, plasma purine concentrations may be raised by increased production or decreased renal clearance of these compounds. ${ }^{5}$ Plasma hypoxanthine concentrations could have been increased by the hyperpermeability of the cell membrane or decreased renal clearance of hypoxanthine. But concentrations of creatine kinase and creatinine were similar before and after the exercise test (not shown) and other reports ${ }^{56}$ suggest that the increase in the concentration of hypoxanthine was probably caused by increased synthesis, with some contribution from impaired renal clearance.

The possible mechanisms of excess purine degradation in the skeletal muscle after submaximal treadmill exercise are: $(a)$ an "absolute" disturbance of the supply of ATP caused by deficient glycogenolysis or glycolysis; or (b) a "relative" disturbance in the supply of ATP - that is, the demand for ATP exceeds its supply. Where there was an absolute disturbance, such as in glycogen storage disease, there was no accompanying increase in lactate ${ }^{3}$; but a relative disturbance, such as in hyperthyroidism, was associated with an increase in lactate. ${ }^{4}$ In our study, an increase in plasma hypoxanthine was accompanied by an increase in plasma lactate. Thus excess purine degradation in our patients could have been the result of a "relative" disturbance in the supply of ATP-that is when the demand for ATP exceeded its supply during exercise, because during submaximal exercise glycolysis became anaerobic and much less efficient than aerobic glycolysis at generating ATP. Excess purine degradation was also accompanied by an increase in plasma noradrenaline. In an earlier study there was an inverse correlation between plasma noradrenaline and pulmonary artery oxygen saturation, showing that the response of the sympathetic nervous system changed to meet the oxygen demand of working skeletal muscle. ${ }^{7}$ The excess purine degradation in our patients might be related to the "relative" disturbance in the supply of ATP in the working muscle caused by the cell energy crisis ${ }^{8}$ precipitated by hypoxia during exercise. The rise in plasma hypoxanthine concentrations may be a good index of a deficiency of energy in the cell during exercise caused by insufficiency of the peripheral circulation in patients with congestive heart failure.

This hypothesis accords with the finding that plasma concentrations of hypoxanthine were much less likely to be high in patients treated with angiotensin converting enzyme inhibitors and vasodilators. In one patient a high plasma concentration of hypoxanthine in response to exercise was abolished by percutaneous balloon dilatation of the mitral valve.

Hypoxanthine can cause direct tissue injury. The enzyme xanthine oxidase converts hypoxanthine to xanthine and uric acid. Release of oxygen metabolites during this oxidation process peroxidises the cell membrane. We found that hypoxanthine, a substrate for xanthine oxidase, was increased after submaximal exercise in some patients with congestive heart failure. Could direct tissue injury by increased hypoxanthine lead to arrhythmia? Certainly, exercise induced arrhythmia was more common in the patients with high plasma hypoxanthine concentrations than in those without. The possibility of a relation between increased plasma hypoxanthine and exercise induced arrhythmia should be investigated.

This work was supported by the Keijinkai Foundation. We thank Dr Akira Takeda for advice.

1 Sutton JR, Toews CJ, Wards GR, Fox IH. Purine metabolism during strenuous muscular exercise in man. Metabolism during strenuou

2 Atkinson DE. Cellular energy metabolism and its regulation. London: Academic Press, 1977.

3 Mineo I, Kono N, Hara N, et al. Myogenic hyperuricemia: a common pathophysiologic feature of glycogenolysis types III, V, and VII. N Engl J Med 1987;317:75-80.

4 Hisatome I, Ishiko R, Takeuchi T, et al. Excess purine degradation in skeletal muscle with hyperthyroidism Muscle Nerve 1990;13:558-9.

5 Grum CM, Simon RH, Dantzker DR, Fox IH. Evidence of adenosine triphosphate degradation in critically-il patients. Chest 1985;88:763-7.

6 Ketai LH, Simon RH, Kreit JW, Grum MC. Plasma hypoxanthine and exercise. Am Rev Respir Dis 1987, 136:98-101.

7 Hansen JF, Christensen NJ, Hesse B. Determinations of coronary sinus noradrenaline in patients with ischemic heart disease. Coronary sinus catecholamine concentratrine concentration pulmonary artery oxygen end-diastolic pressure.
Cardiovasc Res 1978;12:415-21.

8 Fox IH, Palella TD, Kelley WN. Hyperuricemia: a marker for cell energy crisis. $N$ Engl J Med 1987;317:111-2. 